\title{
Thoracoscopic lobectomy for lung cancer with unilateral absence of pulmonary artery
}

\author{
Daisuke Matsumoto, MD, ${ }^{\mathrm{a}}$ Hiroaki Toba, MD, PhD, ${ }^{\mathrm{a}}$ Kazuya Kondo, MD, $\mathrm{PhD},{ }^{\mathrm{b}}$ and \\ Akira Tangoku, MD, $\mathrm{PhD},{ }^{\mathrm{a}}$ Tokushima, Japan
}

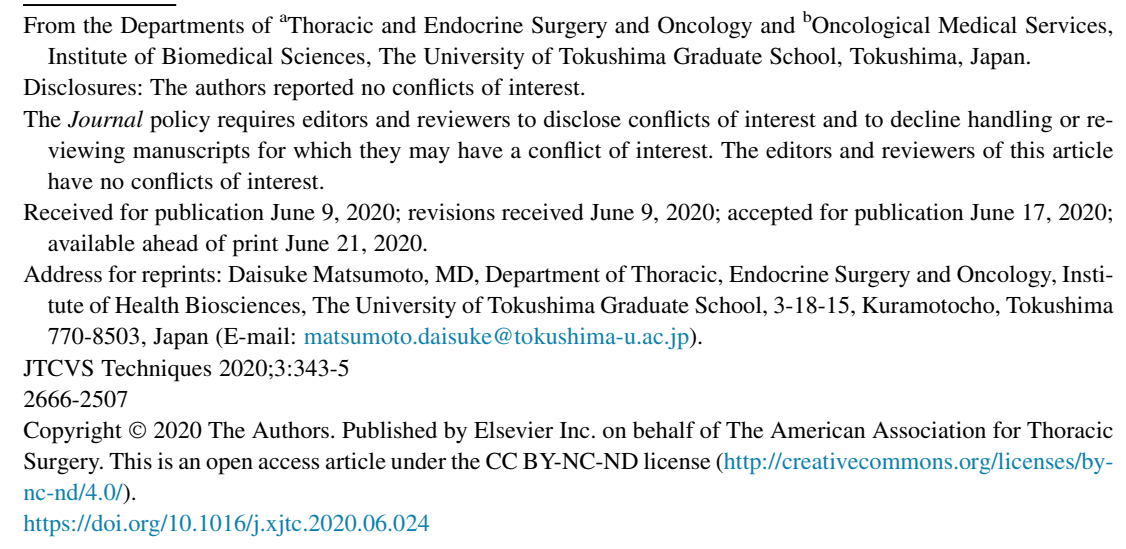

Video clip is available online.

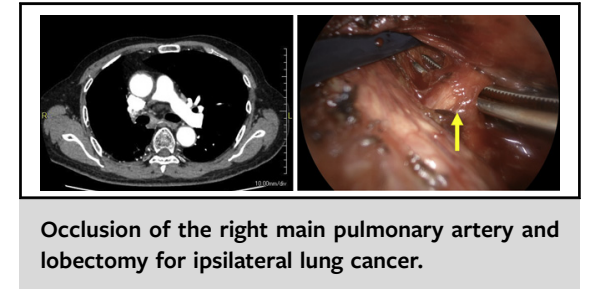

CENTRAL MESSAGE

We report a case of thoracoscopic lobectomy for lung cancer with ipsilateral absence of pulmonary artery. One must be mindful of bleeding due to high blood flow from collateral circulation.

See Commentaries on pages 346 and 348.
Unilateral absence of the pulmonary artery (UAPA) is a rare congenital cardiovascular malformation often associated with congenital cardiac anomalies; however, it can also present alone. Owing to the absence of the pulmonary artery to supply blood to the affected lung, the lung receives its blood supply from the systemic circulation via a collateral artery. We report a case of thoracoscopic lung lobectomy for the treatment of ipsilateral lung cancer with UAPA. Informed consent was obtained from the patient.

\section{CASE DESCRIPTION}

A man in his $80 \mathrm{~s}$ was referred to our department for the treatment of non-small-cell lung cancer (cT1cN0M0, stage IA3). Chest radiography showed a reticular shadow and lung shrinkage only on the right side (Figure 1, A); therefore, we performed ventilation and blood flow scintigraphy. However, no blood flow to the right lung was detected. An enhanced computed tomography (CT) scan revealed a $28-\mathrm{mm}$ nodule in the right lower lobe and a reticular shadow in the right lung (Figure $1, B$ ). In addition, the right main pulmonary artery was completely occluded (Figure 1, $C$ and $D$ ), and the growth of blood vessels supplying the lungs was derived from the systemic circulatory system. Based on these findings, we diagnosed UAPA. Furthermore, no cardiovascular malformation was seen on the CT scan or ultrasonic cardiography.

We discussed the patient's treatment plan at a conference of surgeons, physicians, and radiologists and concluded that radiation therapy should be excluded owing to the presence of interstitial pneumonia in the base lung. We performed thoracoscopic lobectomy for ipsilateral lung cancer. We placed the first port on the midaxillary line of the eighth intercostal space based on transthoracic ultrasonography, which revealed multiple arteries flowing from the anterior chest wall to the visceral pleura and demonstrated decreased mobility of the lung. The right upper and middle lobes were adhered to the chest wall with increased vascular proliferation (Figure 2, $A$ and $B$ ).

The bleeding tendency was high owing to an increased number of thick collateral branches from the bronchial and esophageal arteries into the lung. We initially treated these arteries with a vessel sealing device; however, 

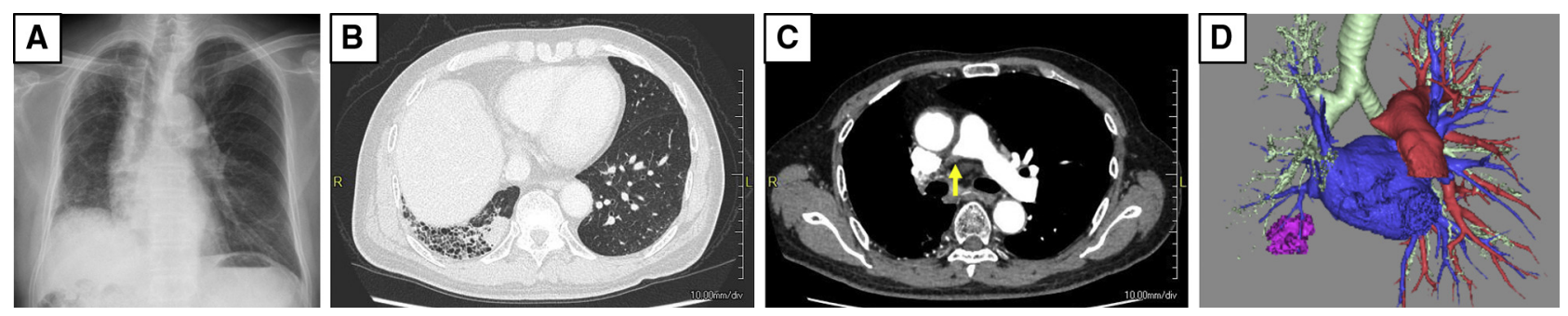

FIGURE 1. A, Chest radiograph showing a reticular shadow and lung shrinkage on the right side. B, Chest computed tomography scan revealing a 28 -mm nodule at the bottom of the right lung, with interstitial changes present only on the right lung. $\mathrm{C}$ and $\mathrm{D}$, The right main pulmonary artery is completely occluded (arrow).

multiple collapses occurred after sealing, and thus we added clips for these bleeds. The lung showed fibrotic changes and fragility. We identified the scarred pulmonary artery at the interlobar surface of the middle and lower lobes and dissected it using a linear stapler (Figure 2, C). We successfully completed the right lower lobectomy and inferior mediastinal lymphadenectomy (Video 1).

\section{DISCUSSION}

Previous studies have indicated that the prevalence of isolated UAPA ranges from 1 in 150,000 to 1 in 300,000 adults. ${ }^{1,2}$ Three ipsilateral and contralateral cases each of UAPA have been reported with lung cancer. The present report demonstrates the following surgical considerations for an ipsilateral case: (1) because the resected lung did not contribute to oxygenation, resection did not adversely affect or improve respiration; (2) collateral circulation developed from the chest wall and mediastinum; (3) the lungs were fragile because of interstitial or emphysematous changes; and (4) the scarred pulmonary artery on the surgical side was difficult to identify.

Several considerations guided our operative strategy. First, the surgical procedure involved partial resection, lobectomy, and pneumonectomy. Partial resection is the safest technique, although less curative. Pneumonectomy may be better for oxygenation; however, there is an increased risk of bleeding from the collateral circulation. Lobectomy should be considered to strike a balance between curability and safety. Second, we considered certain measures for collateral circulation. The most important measure was to avoid damage to the lungs, which was also the most effective measure for lung fragility. We used a vessel sealing device to detach the adhesions and dissect the mediastinal lymph nodes carefully. We considered preoperative embolization but did not perform it because of concerns about insufficient blood flow in the residual lung after lobectomy. There has been no report on the performance of preoperative embolization. Furthermore, we identified the scarred common basal pulmonary artery by dissection, considering a normal anatomy, and obtained confirmation based on the positional relationship with the basal bronchus. We went through the fissure to cut the bronchus and then found the pulmonary artery. In previous reports, the pulmonary artery was identified and described as a fibrous tissue in 2 of 3 cases $^{3,4}$; however, the reports did not describe the method of dissection. We dissected the scarred pulmonary artery with a linear stapler, but it might be possible to dissect the bronchus or lung parenchyma.

In conclusion, ipsilateral lung cancer with UAPA is extremely rare. Careful attention to fragile lungs and
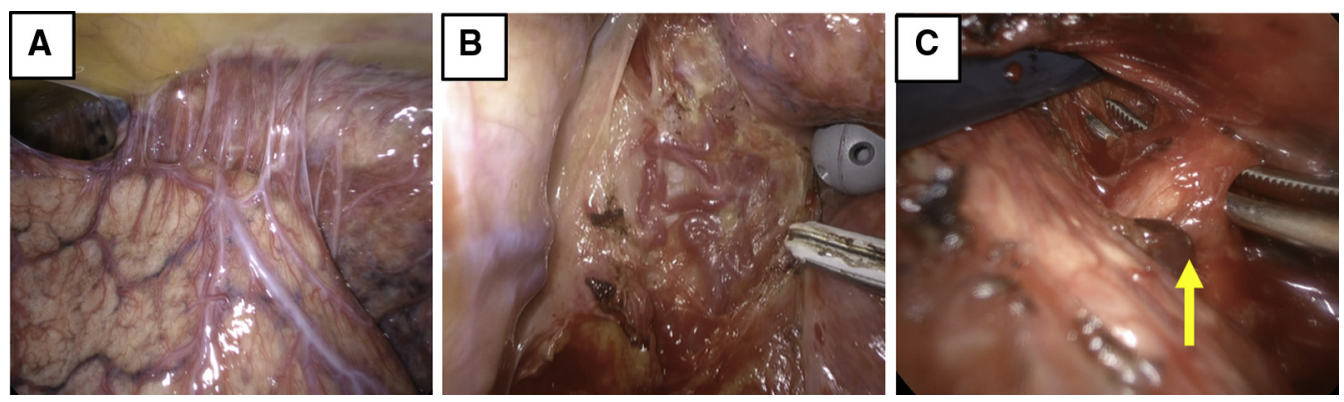

FIGURE 2. A and B, Thoracoscopy showing numerous collateral circulations from the chest wall and mediastinum. C, The scarred pulmonary artery on the interlobar surface of the middle and lower lobes (arrow). 


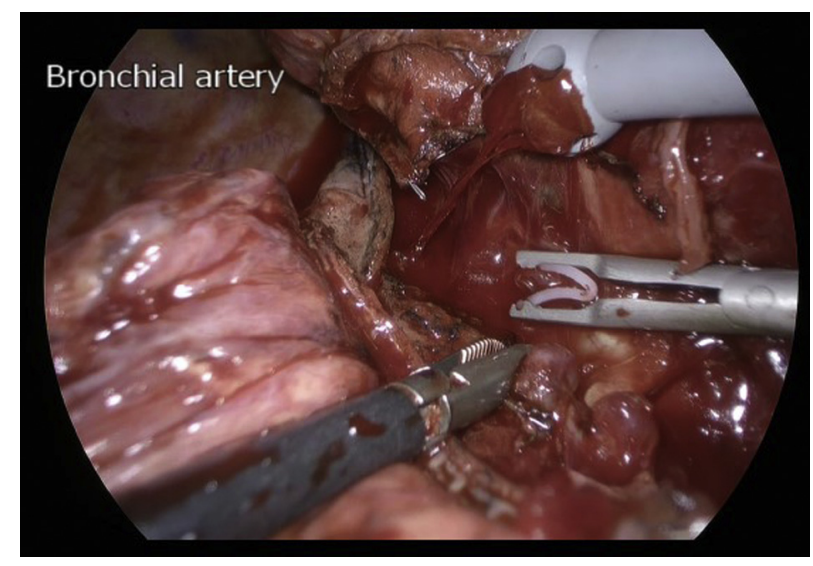

VIDEO 1. Thoracoscopic lung lobectomy to treat ipsilateral lung cancer with unilateral absence of the pulmonary artery. Video available at: https://www.jtcvs.org/article/S2666-2507(20)30302-3/fulltext. collateral arteries allows for a safe procedure using videoassisted thoracic surgery.

\section{References}

1. Kruzliak P, Syamasundar RP, Novak M, Pechanova O, Kovacova G. Unilateral absence of pulmonary artery: pathophysiology, symptoms, diagnosis and current treatment. Arch Cardiovasc Dis. 2013;106:448-54.

2. Bouros D, Pare P, Panagou P, Tsintiris K, Siafakas N. The varied manifestation of pulmonary artery agenesis in adulthood. Chest. 1995;108:670-6.

3. Makdisi G, Edell ES, Maleszewski JJ, Molina JR, Deschamps C. Pulmonary artery agenesis associated with emphysema and multiple invasive non-small-cell lung cancers. Ann Thorac Surg. 2015;99:2192-5.

4. Watanabe Y, Shibuya J, Handa M, Watanabe T, Notsuda H, Saito R, et al. Unilateral absence of the right pulmonary artery accompanied by right lung cancer. Ann Thorac Surg. 2015;100:1113. 